Original Research Paper

\title{
Improving Routing Discovery Based Velocity-Aware Probabilistic Using AODV with Link Prediction in Mobile Ad- hoc Networks
}

\author{
Zaid Bassfar \\ Department of Information Technology, University of Tabuk, Tabuk, Saudi Arabia
}

\author{
Article history \\ Received: 13-05-2017 \\ Revised: 30-06-2017 \\ Accepted: 01-08-2017
}

Email: zbassfar@ut.edu.sa

\begin{abstract}
The current process associated with enhancing the QoS in routing for MANETs has reserved a high importance due to the limitation of available route discovery in dynamic topology. The conventional methods of route discovery schemes in MANETs are commonly designed in order to estimate the number of nodes in routes using the standard probabilistic models. Such models are utilized by estimating the flood in a network using the RREQ packets. However, the current development in mobility, frequent breakages become more common which as a result lead to relocate the properties of same routes. As such, the network may experience additional demands for channel contention and high packets collision rate. For this, we proposed a new scheme for enhancing the routing discovery based velocity-aware probabilistic using AODV properties with link prediction in MANETs. The result of the new scheme was found to outperform the old one in which it offers better ROH, throughput and delay time. This study provides an important insight for predicting the routing failure in a network when using the velocity-aware probabilistic scheme alone.
\end{abstract}

Keywords: MANETs, AODV Performance, RREQ, Link Prediction in MANET, Velocity-Aware Probabilistic

\section{Introduction}

The current complexity in network topologies of Mobile Ad-hoc Networks (MANETs) is typically driven by the frequent changes occurs when nodes transfer freely with no restriction in terms of directions. The process associated with routing data and packet dissemination is somehow problematic that usually found when nodes modes in high speed and different directions. Due to this, the established links between network nodes can be expired frequently (Ghali et al., 2014; Karami, 2015). As such, it deemed essential to discover potential break in wireless links between nodes which commonly deals with flooding the network with Route Requests (RREQs), Route Replies (RREPs) packets. This problem can be illustrated when the route discovery phase of the Ad Hoc On-demand Distance Vector Routing Protocol (AODV) protocol attempt to locate the optimal route to the required destination by replacing the network with RREQ control packets (Perkins et al., 2007). Based on this, a link breakage is likely to occur during the movement of nodes in the route because it may contain unstable nodes, which as a result may affect the overall network performance. Therefore, previous studies recommended solving current link breakages in the node in order to enhance the network performance (Chuang et al., 2012).

\section{Review of Literature}

Previous studies like Xu and Li (2014) illustrated the needs for providing a better route detection algorithm that ensure link stability and energy aware routing with the consideration of tradeoff strategy in AODV. They highlighted the potential of regulating the current protocols in order to offer a better stability in route discovery based on link stability and node energy information. This can be achieved by the immediate prediction of the link breakage in route maintenance. Besides, the routing takes a route stability-hop count tradeoff strategy to select routes with high stability and low hop count. For example, Yadav et al. (2015) proposed a novel way to stringing the signal based link availability prediction in AODV routing. The method works on estimating the link 
breakage time and further warns the other nodes about the link breaks in the route. Such information can be used to provide the local route repair or new route discovery much earlier than the route breakage. Such method is believed to reduces the data packet losses as well as end-to-end delay. In addition, Qi et al. (2015) developed a multi-path routing protocol in AODV based on estimating the energy of available nodes. EM-The workability of the proposed method is typically based on obtaining nodes energy by upgrading the route discovery and route maintenance process of AODV, calculates the path of comprehensive energy derived path priority by routes total hops and nodes energy to format the multi-path routing mechanism. Such process is assumed to stabilize the nodes energy bound and balancing nodes data forwarding to postpone network lifetime. Yamaguchi et al. (2016) proposed the development of routing methods that consider the traffic load balancing and QoS based on the AODV and Optimized Link State Routing (OLSR) protocols. The proposed method consists of estimating the traffic load from the routing in order to establish route without any repeater nodes having high traffic load by monitoring network traffics on each node. The authors claimed that such combination helps to increase the overall network performance. Abbas et al. (2015) proposed a fuzzy-based approach to help improving the AODV reactive routing performance by allocating the trusted nodes in order to establish the route between the source and destination nodes. Authors used nodes' parameters, such as residual energy, node mobility and number of hop counts, through a fuzzy inference system to facilitate the process of estimating the value of the node trust level, which can be used as a metric to construct an optimal path from source to destination. The simulation outcomes showed an improvement in the network performance when using the proposed method than the traditional AODV routing protocol. On the other hand, Zhang et al. (2014) asserted that predicting link breakage in a network with heavy traffic is a problematic issue in which the standard prediction methods are mostly works at reducing the amount of overhead in the route discovery by reducing the amount of RREQ packets. As a result, it is believed that such reduction would results in limiting the number of the underlying paths that route discovery can find. Based on this, the process for predicting the stablest path for data packets transmission is a problematic. Other scholars have proposed different solutions to overcome the link prediction problems of the route in AODV, for example Khan et al. (2011) proposed that node should cancels its retransmission if it has a density degree above a predefined density threshold. Another example in Khalaf et al. (2015) that utilized the probabilistic concept to develop new probabilistic routing schemes, namely Simple Velocity Aware Probabilistic (SVAP) and Advanced Velocity Aware Probabilistic (AVAP) route discovery schemes for the aim of solving the frequent link breakages problem. However, these methods do not consider connectivity and power limitation issues in ad-hoc networks. In addition, it is evident that nodes in high-density networks is usually turned off which results in maximizing transmission range and increase energy efficiency. Hence, this study proposed modifying the current AVAP named AODV NEW where each node has a table of adjacent nodes that can provide a significant redundant retransmission, causing high channel usage and packet collisions in the network. Based on these, it become evident that the link breakage usually occurs when a certain node drops from the network in which the link between the dropped node and its neighbors is no longer valid. However, the process associated with allocating a new routing discovery for the dropped node imposes extra overhead on the network. This has been acknowledged to demands extra time for packets to be transferred and causes the broadcast storm problem. Therefore, many probabilistic routing methods has been developed to reduce the broadcast storm problem in AODV protocol which usually done by stopping nodes from participating in route discovery based on some predefined thresholds. This study aims at achieving the following:

- Develop a routing discovery scheme based on the properties of AVAP to reduce the link breakage rate in a network

- Compare the network performance of the proposed algorithm with the conventional AODV and the modified AVAP schemes

\section{Method}

The research design of this study consists of estimating the distance of the produced error or break in route direction in which a guess based matrix will be used to determine the shortest route for transferring packets. In addition, an adjustment of that path will be determined based on the metric $\mathrm{m}$, which must be less than the distance of the predicted error or break as shown in Fig. 1. The process started with identifying the epsilon value based on the initial guess in the available nodes. This is usually estimated by the second of time that passes in which the velocity as a result may gains additional $0.5 \mathrm{~m} / \mathrm{s}$. In this study, it is assumed that increase in the velocity is mostly contributed to the number of seconds that have passed. The $b$ value will be then adjusted in order to estimate the metric value of $\mathrm{m}$ for comparison purposes. 


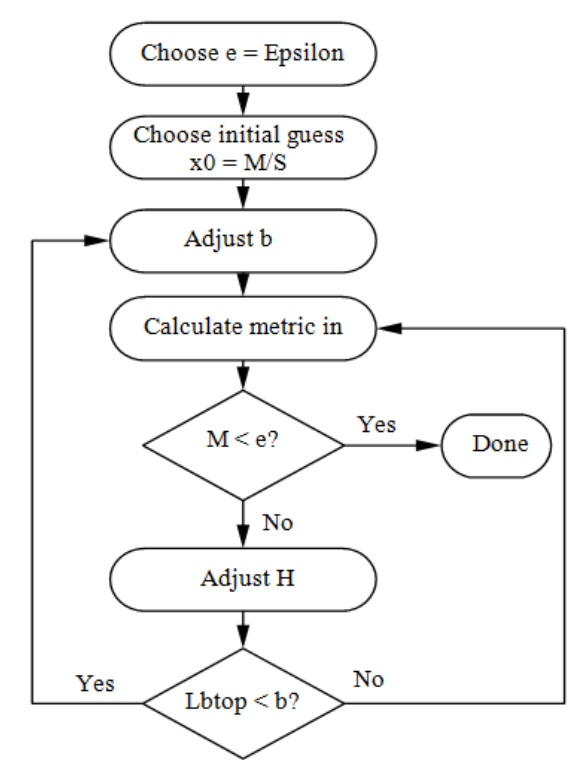

Fig. 1. Research design

We attempted to provide a better performance for AODV routing protocol of the method mentioned in previous works of Khalaf et al. (2015). This was achieved by combining the technique of velocity-aware probabilistic route discovery schemes for high mobility Ad hoc networks proposed by Khalaf et al. (2015) with the with link prediction in Mobile Adhoc Networks proposed by Yadav et al. (2015). The reason for doing so back to the feasibility of link prediction technique in advancing the velocity-aware probabilistic by estimating any potential link breakage among nodes and warns the other nodes about it in the route. By doing so, it is believed that routing discovery based velocity aware probabilistic can send request for local route repair or new route discovery is initiated much earlier than the route breakage.

We used NS2 for simulating the proposed combination. In this work, we estimated the value of power and time of the last three packets as follow:

void AMV:: Update Power Time(double power Val, double time) \{

$$
\begin{aligned}
& \text { lastPower[0] = lastPower[1]; } \\
& \text { lastPower[1] = lastPower[2]; } \\
& \text { lastPower[2] = powerVal; } \\
& \text { lastTime[0] = lastTime[1]; } \\
& \text { lastTime[1] = lastTime[2]; } \\
& \text { lastTime[2] = time; }
\end{aligned}
$$

\}

When the value of last received power is lower than the previous power $(\mathrm{p} 1>\mathrm{p} 2>\mathrm{p} 3)$, then we can predict the status of bad routing path. The status was then reserved in order to determine the time and power value for the node. This helped us to efficiently estimate the status of routing path accurately. On the other hand, in the event that the received status is bad, then a REPAIR message should be sent to the next node as illustrated below:

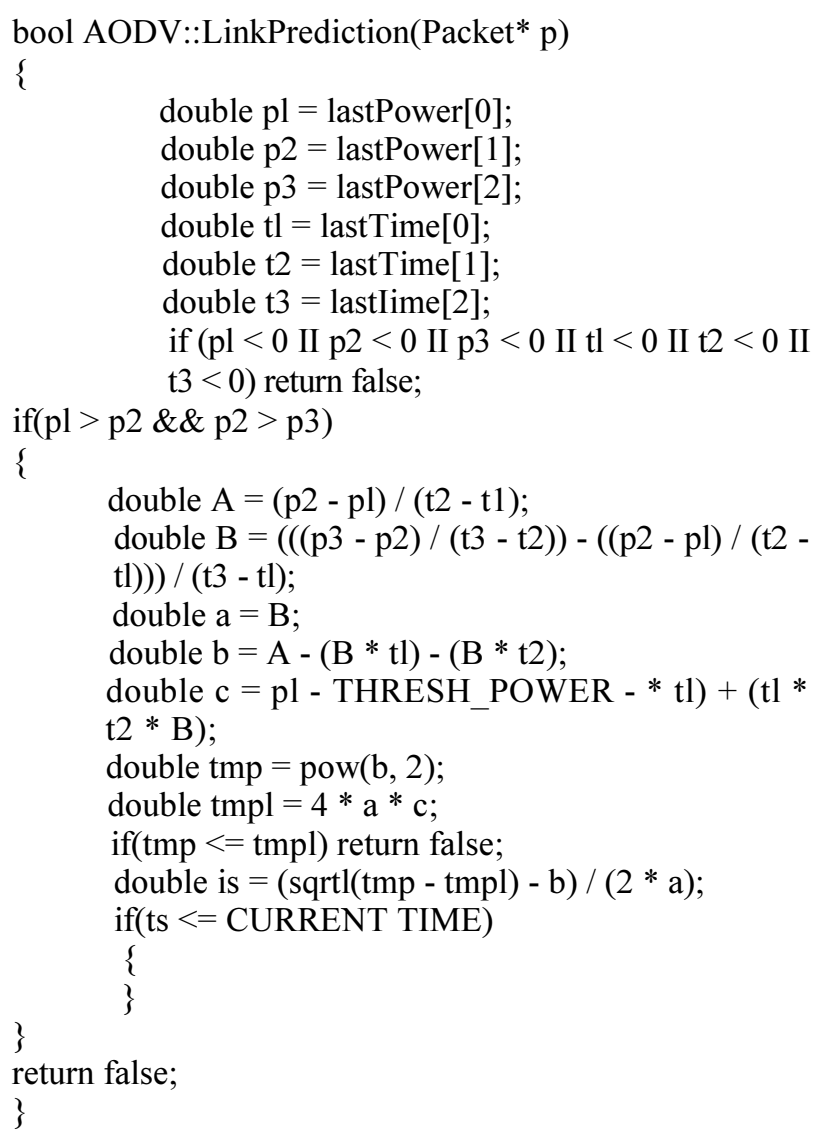

Using this technique can enable us to repair the routing path before the routing path is failed. Therefore, the performance of AODV routing algorithm can be further improved as shown below:

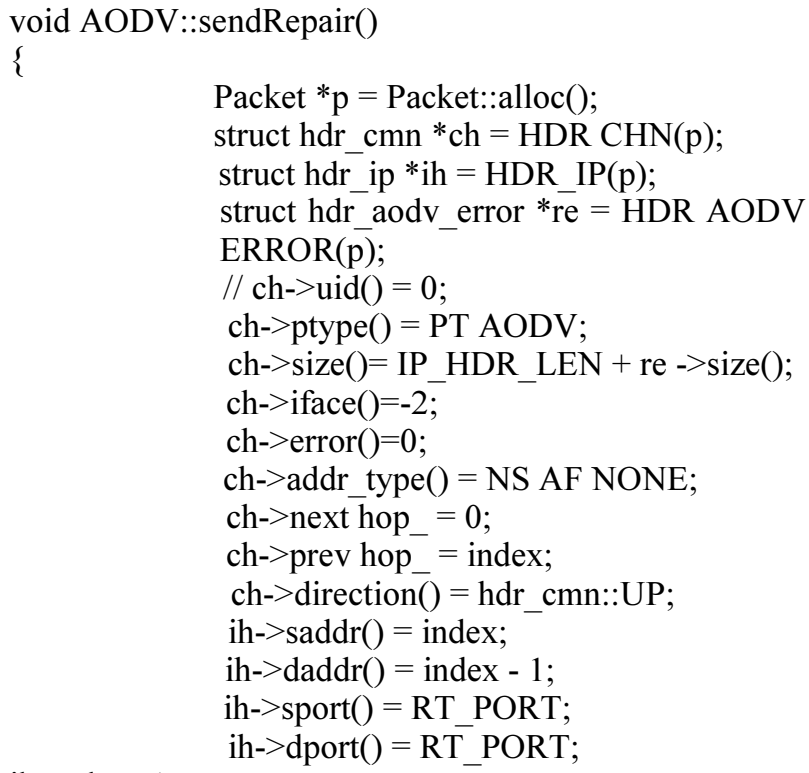


Table 1. Simulation parameters

\begin{tabular}{ll}
\hline Parameter & Value \\
\hline Transmitter range & 250 \\
Bandwidth & $2 \mathrm{M}$ bit \\
Interface queue length & $50 \mathrm{messages}$ \\
Simulation time & $900 \mathrm{sec}$ \\
Pause time & $0 \mathrm{sec}$ \\
Packet size & $512 \mathrm{bytes}$ \\
Topology size & $1000 \times 1000 \mathrm{~m}^{2}$ \\
Nodes speed & $5-100 \mathrm{~m} / \mathrm{sec}$ \\
Number of node & $20 / 200$ nodes \\
Number of connection & 20 \\
Mobility model & Random way-point \\
\hline
\end{tabular}

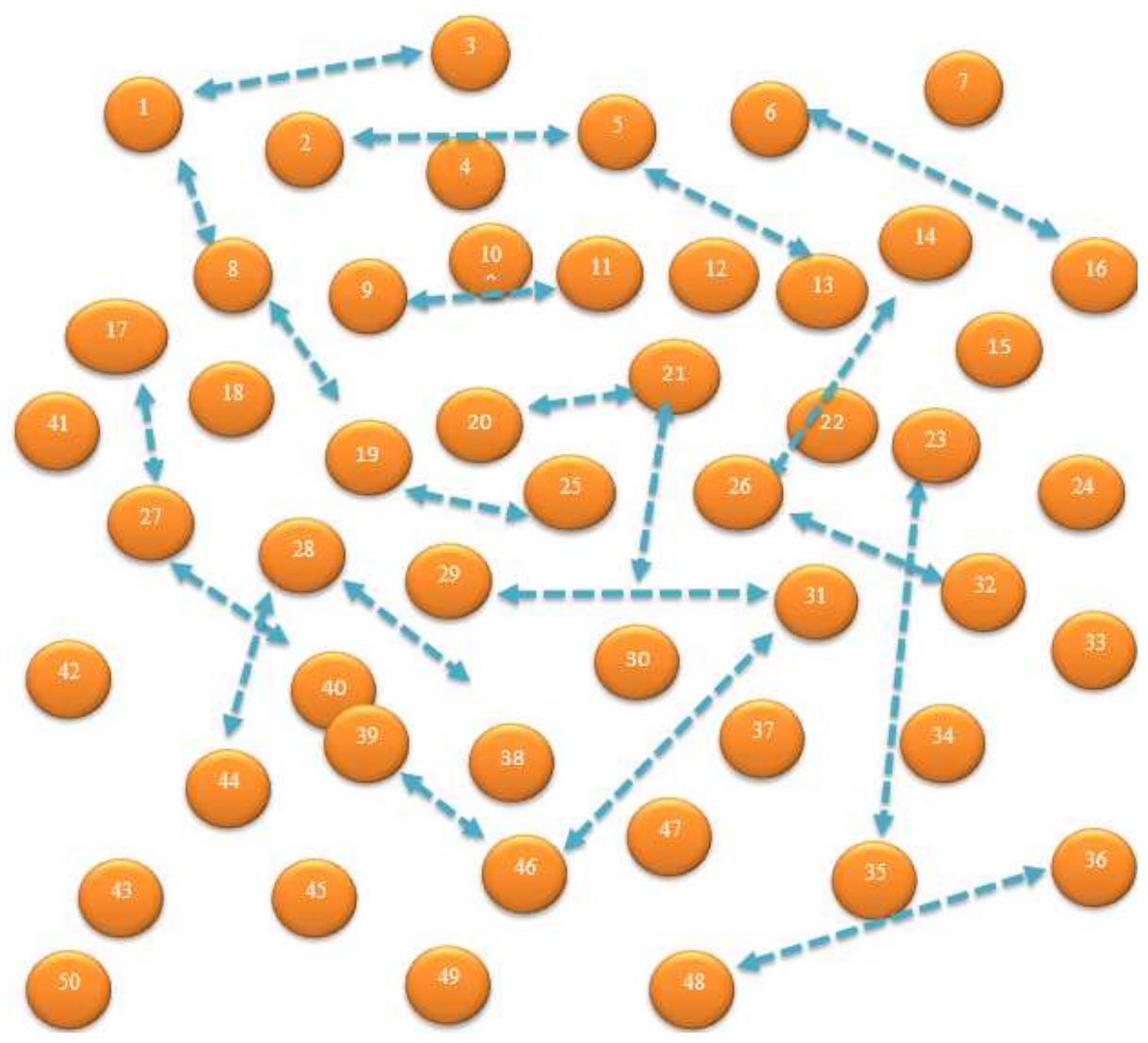

Fig. 2. MANET topology

\section{Topology}

It is evident that the design topology of network in MANET is unorganized and varies greatly from one second to the next. Hence, the use of randomly distributed topology is sufficient in this study. Figure 2 shows an example of the MANET topology.

Varieties of different scenarios were run in the NS2 simulation. Table 1 below shows the baseline parameter set.

\section{Performance Metrics}

Three performance metrics were used in this study named Routing Overhead $(\mathrm{ROH})$, throughput and delay time. The $\mathrm{ROH}$ was measured based on the estimation of total number of RREQ packets generated by each node and rebroadcasts during the period of the simulation time. The throughput value was measured based on the total of all delivered packets to destination by the total time as per $\mathrm{KB} / \mathrm{Sec}$. While the delay time was measured by estimating the average period time when the source transmits data packet along with the average period time when it's delivered to the destination.

\section{Results}

The obtained results based on $\mathrm{ROH}$, throughput and delay time were extracted and compared with the original method of velocity-aware probabilistic route 
discovery schemes for high mobility Ad hoc networks proposed by Khalaf et al. (2015). Figure 3 shows the changes in nodes speed which is incurred by the proposed scheme. The result showed that our scheme delivers better $\mathrm{ROH}$ than the original scheme in which when the number of nodes increase, it results in increasing the number of RREQ packets. In addition, the maximum speed of nodes when using the new scheme was improved by consuming less packets per second. Such result can be reasoned to that our scheme treat larger number of the forwarded nodes which as a result process larger number of nodes. This result opens new avenues to studies on route discovery schemes in using velocity-aware probabilistic based on the estimation of time and power needed to process nodes. It can also add to the work of Jain and Sharma (2014) in which the filtering forwarding scheme can be enhanced based on the velocity-aware probabilistic of excessive RREQs resulted from the node per unit time. It may also contributes to the study of Reina et al. (2014) by reducing the redundancy of routing packets during the discovery phase of the reactive routing protocols for MANETs.

Figure 4 presents the throughput result of our scheme with link prediction compared to the previous scheme of velocity-aware probabilistic. From the result, it can be noticed that the proposed scheme resulted in a better throughput value than the old one. This result can help previous studies like Pandey et al. (2014) to increase the performance of AODV in different network sizes. This include combining the feature of velocity-aware probabilistic with travelling salesman problem in order to increase number of nodes as it is known that routing protocols makes an important task for improving QoS. The obtained result can also be used to enrich findings of Abedi et al. (2009) by using velocity-aware probabilistic to reduce the time taken in the route discovery phase by restricting neighbor's distance and number of discovered routes.

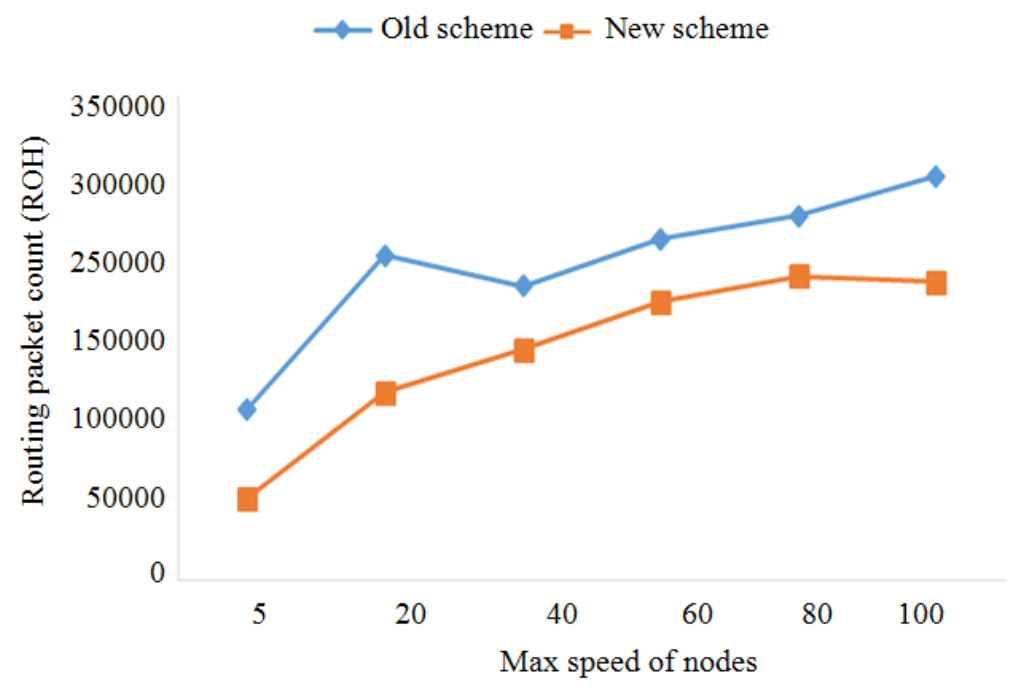

Fig. 3. ROH result of the proposed scheme compared with previous scheme

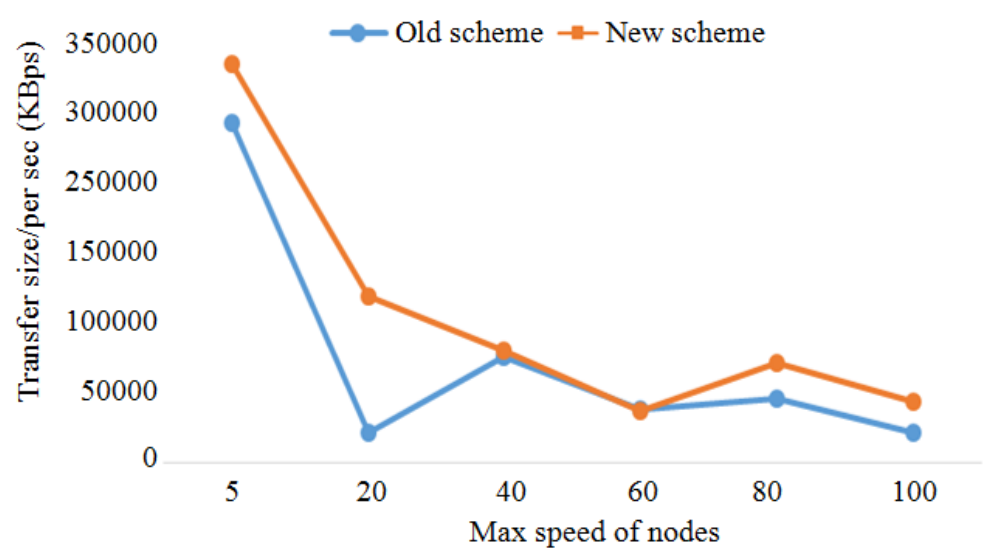

Fig. 4. Throughput result of the proposed scheme compared with previous scheme 


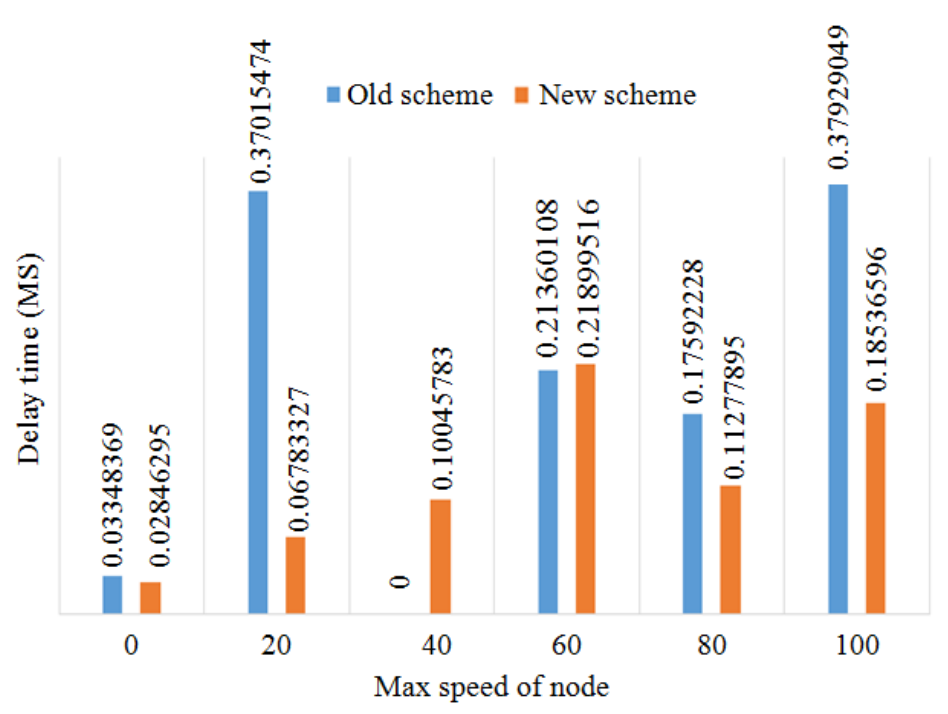

Fig. 5. Delay time result of the proposed scheme compared with previous scheme

The same is true when looking at the result of delay time in Fig. 5. It is obvious from the figure that delay time using the proposed scheme is less than it is in the old scheme. This can be due to that when the link prediction decreased the propagation delay when the network density increases. Such finding can be relevant to the development of sufficient mechanism for estimating nodes' time, which may contribute to Jhaveri et al. (2012) to which the proposed scheme can obtain unusual node from the routing information or misbehaving neighbor node, which, include routing packets and pass routing information.

\section{Conclusion}

We proposed a new scheme based on probabilistic broadcast scheme for MANETs, with link prediction mechanism. The new scheme was found to overcome the current limitation in network performance of the original probabilistic broadcasting one. The simulation result consists of comparing the $\mathrm{ROH}$, throughput and delay time proved the feasibility of the new scheme in different operating conditions and scenarios. Our findings revealed that the new scheme based on the use of link prediction into the velocity-aware probabilistic scheme has improved performance of routing discovery in MANETs. This study was limited to the link prediction mechanism with boarder emphasis on the network performance. This study considered adjusting the AODV network parameters in order to suite the simulation. NS2 was used for simulating the proposed algorithm and comparing the result of the proposed mechanism with previous methods. This study provides an important insight for predicting the routing failure in a network. This study argues that enhancing the Weighted Probabilistic-Persistence Broadcasting (WP-PB) scheme each link $(I, J)$ is associated with a probability $P(I, J)$ to develop better, more stable probabilistic protocol.

\section{Acknowledgement}

The author would like to acknowledge University of Tabuk for the financial support under research project number S-1437-0255.

\section{Funding Information}

This work was financially supported by University of Tabuk under research project number S-1437-0255.

\section{Ethics}

The author confirm that this manuscript has not been published elsewhere and that no ethical issues are involved.

\section{References}

Abbas, N.I., M. Ilkan and E. Ozen, 2015. Fuzzy approach to improving route stability of the AODV routing protocol. EURASIP J. Wireless Commun. Netw.

Abedi, O., R. Berangi and M.A. Azgomi, 2009. Improving route stability and overhead on AODV routing protocol and make it usable for Vanet. Proceedings of the 29th IEEE International Conference on Distributed Computing Systems Workshops, (CSW' 09), pp: 464-467.

Chuang, P.J., P.H. Yen and T.Y. Chu, 2012. Efficient route discovery and repair in mobile Ad-hoc networks. Proceedings of the IEEE 26th International Conference on Advanced Information Networking and Applications (INA' 12), pp: 391-398.

Ghali, C., G. Tsudik and E. Uzun, 2014. Network-layer trust in named-data networking. ACM Sigcomm Comput. Commun. Rev., 44: 12-19. 
Jain, H.R. and S.K. Sharma, 2014. Improved energy efficient secure multipath AODV routing protocol for MANET. Proceedings of the International Conference on Advances in Engineering and Technology Research (ETR' 14), pp: 1-9.

Jhaveri, R.H., S.J. Patel and D.C. Jinwala, 2012. Improving route discovery for AODV to prevent blackhole and grayhole attacks in MANETs. Infocomp J. Comput. Sci., 11: 1-12.

Karami, A., 2015. ACCPndn: Adaptive congestion control protocol in named data networking by learning capacities using optimized time-lagged feedforward neural network. J. Network Comput. Applic., 56: 1-18. DOI: 10.1016/j.jnca.2015.05.017

Khalaf, M.B., A.Y. Al-Dubai and G. Min, 2015. New efficient velocity-aware probabilistic route discovery schemes for high mobility Ad hoc networks. J. Comput. Syst. Sci., 81: 97-109.

Khan, I.A., S.A. Madani, W. Anwar and K. Hayat, 2011. Location based dynamic probabilistic broadcasting for MANETs. World Applied Sci. J., 13: 2296-2305.

Pandey, T., S. Solanki and R. Dubey, 2014. Improved performance of AODV routing protocol with increasing number of nodes using traveling salesman problem. Int. J. Comput. Applications.

Perkins, C., E. Belding-Royer and S. Das, 2007. Ad hoc On-Demand Distance Vector (AODV) routing.
Qi, X., Q. Wang and F. Jiang, 2015. Multi-path routing improved protocol in AODV based on nodes energy. Int. J. Future Generation Commun. Networking, 8: 207-214.

Reina, D., S. Toral, P. Johnson and F. Barrero, 2014. Improving discovery phase of reactive ad hoc routing protocols using Jaccard distance. J. Supercomputing, 67: 131-152.

$\mathrm{Xu}, \mathrm{B}$. and Y. Li, 2014. A novel link stability and energy aware routing with tradeoff strategy in mobile ad hoc networks. J. Commun., 9: 706-713.

Yadav, A., Y. Singh and R. Singh, 2015. Improving routing performance in AODV with link prediction in mobile Adhoc networks. Wireless Personal Commun, 83: 603-618.

Yamaguchi, K., K. Mukaiyama, T. Akiyama, T. Kobayashi and $\mathrm{H}$. Matsue, 2016. Performance analysis of routing methods based on OLSR and AODV with traffic load balancing and QoS for Wi-Fi mesh network. Proceedings of the International Conference on, Information Networking (COIN' 16), pp: 114-119.

Zhang, X., K. Chen, Y. Zhang and D.K. Sung, 2014. A probabilistic broadcast algorithm based on the connectivity information of predictable rendezvous nodes in mobile ad hoc networks. Proceedings of the 23rd International Conference on, Computer Communication and Networks (CCN' 14), pp: 1-6. 